\title{
Effets de la salinité et de la dureté de l'eau sur la toxicité des métaux vis-à-vis de Daphnia magna Strauss
}

\author{
S. Semsari ${ }^{1}$ \\ A. Haït-Amar ${ }^{2}$
}

Mots-clés : Daphnia, bioessais, toxicité, conditions expérimentales, salinité, dureté, métaux lourds.

L'effet de la salinité $\left(0,3-0,45\right.$ et 2,45 g.L. $\left.\mathrm{L}^{-1}\right)$ et de la dureté $\left(250-500\right.$ g.L $\mathrm{L}^{-1}\left(\mathrm{CaCO}_{3}\right)$ sur la toxicité de $\mathrm{Al}, \mathrm{Cu}, \mathrm{Cr}, \mathrm{Cd}, \mathrm{Ni}$, $\mathrm{Zn}$ vis-à-vis de Daphnia magna est étudiée en laboratoire. Les concentrations en ions métalliques varient entre 0,01 et $25 \mathrm{mg} . \mathrm{L}^{-1}$. Les résultats obtenus à $20^{\circ} \mathrm{C}$ montrent que la toxicité mesurée en CI50 $24 \mathrm{~h}$ et CI50 $48 \mathrm{~h}(\mathrm{CI}=$ Concentration d'Inhibition) de $\mathrm{Cd}, \mathrm{Cu}, \mathrm{Zn}$ et $\mathrm{Al}$ augmente avec un accroissement de la salinité ou de la dureté. Par contre, la toxicité de $\mathrm{Ni}$ et $\mathrm{Cr}$ est atténuée malgré l'accroissement de la salinité du milieu. L'augmentation de la dureté entraîne une réduction de la toxicité du chrome.

\section{Effects of water hardness and salinity on metal toxicity to Daphnia magna Strauss}

Keywords : Daphnia, bioassays, toxicity, applied conditions, salinity, hardness, heavy metals.

The effect of salinity $\left(0.3-0.45\right.$ and $\left.2.45 \mathrm{~g} . \mathrm{L}^{-1}\right)$ and hardness $\left(250-500 \mathrm{~g} \cdot \mathrm{L}^{-1}\left(\mathrm{CaCO}_{3}\right)\right.$ on the toxicity of $\mathrm{Al}, \mathrm{Cu}, \mathrm{Cd}, \mathrm{Ni}, \mathrm{Zn}$ and $\mathrm{Cr}$ to Daphnia magna has been investigated in laboratory. The concentrations of metals from 0.01 to $25 \mathrm{mg} . \mathrm{L}^{-1}$ were examinated. The results obtained at $20^{\circ} \mathrm{C}$, show that the toxicity, measured at CI50-24 h and CI50-48 h of Cd, $\mathrm{Cu}, \mathrm{Zn}$ and $\mathrm{Al}$ increases with an increase in salinity or hardness. On the other hand, the toxicity of $\mathrm{Ni}$ and $\mathrm{Cr}$ was affected by the increasing of the salinity. The increase in hardness involves a reduction of chromium toxicity.

\section{Introduction}

Daphnia magna est souvent utilisée comme bioindicateur d'un grand nombre de tests biologiques standardisés en raison de sa sensibilité élevée, de sa grande vitesse de reproduction et de son utilisation simple. Ainsi, divers tests ont été effectués sur sa sensibilité vis-àvis des ions métalliques pouvant être apportés lors de déversements accidentels d'effuents industriels dans les milieux récepteurs (Semsari 1992, Semsari \& Gaïd 1993). Ces tests ont été réalisés en laboratoire, dans des milieux aqueux à faibles salinité et concentration en carbonates.

1. Institut de Chimie Industrielle, Université de Blida, Algérie. 2. Institut de Chimie Industrielle, U.S.T.H.B., B.P. 32, El-Alia, Alger, Algérie.
Par contre, d'autres auteurs ont tenté de simuler le comportement des daphnies dans des eaux de surface caractérisées par une salinité et une dureté élevées, en examinant l'effet de ces paramètres sur la toxicité des ions métalliques vis-à-vis des daphnies.

A ce titre, la plupart des travaux effectués sur l'influence de la dureté de l'eau sur la toxicité des ions métalliques vis-à-vis de Daphnia magna ont surtout concerné les interactions de la dureté et de l'alcalinité (Erickson et al. 1996). Dans leur ensemble, ces résultats sont contradictoires puisque, par exemple, divers auteurs (Zoitko \& Carson 1973, Carter \& Nicholas 1978, Holcombe \& Andrew 1978) ont fait ressortir qu'une augmentation de la dureté et de l'alcalinité tend à diminuer la toxicité du zinc vis-à-vis de Daphnia magna, alors que Berglind \& Dave (1984) ont montré qu'un accroissement de la dureté-alcalinité tend à augmenter les toxicités aiguë et chronique du zinc. 
Contrairement à Winner \& Gauss (1986), Paulauskis \& Winner (1988) expliquent que la toxicité de $\mathrm{Zn}$ diminue avec une augmentation de la dureté car celle-ci en altérerait son mode d'action toxique en intervenant sur la bioaccumulation de $\mathrm{Zn}$.

En plongeant des Daphnies dans un milieu contenant des sels de cadmium et de cuivre, Winner \& Gauss (1986) ont observé que la dureté a un effet sur la toxicité chronique apportée par le cadmium alors que la toxicité du cuivre n'en est pas affectée. Ces auteurs expliquent leurs observations en comparant les constantes de liaison de $\mathrm{Ca}$ et $\mathrm{Mg}$ avec celles de $\mathrm{Cd}$ et de Cu vis-à-vis de la glycine, de l'ATP et de l'acide acétique ; ils concluent que le $\mathrm{Mg}$ et non le $\mathrm{Ca}$, serait en fait, en compétition avec le Cd pour la création de liaisons avec le tissu biologique, diminuant ainsi la toxicité de ce métal.

Les constantes de liaison pour le cuivre sont beaucoup plus élevées que pour le $\mathrm{Cd}$, et les complexes qui en résultent sont beaucoup trop stables pour être inhibés par le $\mathrm{Ca}$ ou $\mathrm{Mg}$.

Concernant l'effet de la salinité, Sprague (1985) suggère que la résistance des micro-organismes est plus importante vis-à-vis des toxiques lorsqu'ils possèdent des capacités osmo-régulatrices.

D'autres auteurs (Sunda \& al. 1978, De Lisle \& Roberts 1988, Voyer \& Mc Govern 1991) ont montré que la salinité modifie l'accumulation et la toxicité aiguë du cadmium, et attribuent l'effet protecteur d'une salinité élevée vis-à-vis de la toxicité, à la complexation des ions métalliques.

Theede (1980) suggère que l'effet de la salinité sur les temps de survie des daphnies est en corrélation étroite avec l'interruption de la régulation osmotique. Il a montré que les temps de survie sont réduits lorsque les salinités sont au-dessus et au-dessous du point isosmotique, correspondant à une augmentation progressive d'une hyper- ou hypo-régulation osmotique qui se situe bien au-delà du point isosmotique.

Dans de nombreuses régions d'Algérie, la qualité souvent saumâtre $\left(3 \mathrm{~g} . \mathrm{L}^{-1}\right)$ ou dure $\left(500\right.$ à $800 \mathrm{mg} . \mathrm{L}^{-1}$ $\mathrm{CaCO}_{3}$ ) des eaux de surface nous a conduit à examiner l'effet de ces paramètres sur la toxicité de quelques ions métalliques ( $\mathrm{Al}, \mathrm{Cr}, \mathrm{Cu}, \mathrm{Ni}, \mathrm{Zn}, \mathrm{Cd})$ vis-à-vis de Daphnia magna et d'observer son comportement en présence d'un tel milieu.

\section{Matériel et méthodes}

\subsection{Méthodologie}

Les daphnies sont maintenues par reproduction parthénogénétique dans un bassin cylindrique de $85 \mathrm{~cm}$ de hauteur et 3,6 de diamètre, préalablement désinfecté, dans lequel sont mélangés : $9 \mathrm{~kg}$ de terre pour sa contenance en sels minéraux; $1,5 \mathrm{~kg}$ de chaux agricole $(\mathrm{Ca}-$ $\mathrm{CO}_{3}$ pur) dont le rôle est d'ajuster le $\mathrm{pH}$ de l'eau, $500 \mathrm{~g}$ d'engrais chimique renfermant respectivement $18 \%$ de potasse et de superphosphate $\left(\mathrm{P}_{2} \mathrm{O}_{5}\right)$ et $12 \%$ d'azote; $7,3 \mathrm{~kg}$ de fumier et une quantité d'eau nécessaire pour couvrir le mélange préparé. Ce mélange est laissé tel quel pendant 10 à 12 jours à une température d'environ $20^{\circ} \mathrm{C}$, avant ensemencement par des daphnies mères. Les daphnies utilisées pour les tests sont élevées dans une enceinte thermostatée à $20 \pm 2^{\circ} \mathrm{C}$, en présence d'une lumière de 500 à 1000 Lux et une photopériode de $16 \mathrm{~h}$ de lumière et $8 \mathrm{~h}$ d'obscurité. L'élevage est effectué dans des cristallisoirs de deux litres. Le milieu est renouvelé trois fois par semaine. La nourriture impliquée est constituée de cellules vivantes d'algue Scenedesmus sp., à raison de $10^{5}$ cellules/daphnie et par jour.

Les biotests considérés sont réalisés dans des tubes de $15 \mathrm{~cm}$ de hauteur et 1,5 de diamètre (ISO, 1989) contenant $10 \mathrm{ml}$ de solution d'essai et 5 daphnies âgées de moins de $72 \mathrm{~h}$. Ces daphnies sont préalablement obtenues sur un jeu de tamis dont les mailles du tamis supérieur $(800 \mu \mathrm{m})$ retiennent les daphnies adultes alors que les mailles du tamis inférieur $(500 \mu \mathrm{m})$ permettent la récupération des jeunes daphnies âgées de moins de $72 \mathrm{~h}$ et qui sont utilisées pour les tests. Pour chaque concentration, 4 tubes à essai sont prévus ainsi qu'un tube témoin dans lequel est introduit un volume d'eau de dilution (ISO, 1989) (Tableau 1) identique au volume des solutions d'essai, avec le même nombre de daphnies. Les tubes sont ensuite placés à l'obscurité dans une étuve thermostatée à $20^{\circ} \mathrm{C}$.

Après des temps d'exposition de 24 et $48 \mathrm{~h}$, la mobilité des crustacés est déterminée en dénombrant les daphnies encore mobiles dans chaque tube.

Après chaque test, le $\mathrm{pH}$ et la concentration en oxygène dissous sont vérifiés. Chaque test est répété trois fois.

Tableau 1. Composition chimique de l'eau de dilution.

Table 1. Chemical composition of the diluted water.

\begin{tabular}{lc}
\hline Eau distillée & Q.s.p $1000 \mathrm{~mL}$ \\
\hline $\mathrm{CaCl}_{2}, 2 \mathrm{H}_{2} \mathrm{O}$ & $11,76 \mathrm{~g} . \mathrm{L}^{-1}(25 \mathrm{~mL})$ \\
$\mathrm{MgSO}_{4}, 7 \mathrm{H}_{2} \mathrm{O}$ & $4,93 \mathrm{~g} . \mathrm{L}^{-1}(25 \mathrm{~mL})$ \\
$\mathrm{NaHCO}_{3}$ & $2,59 \mathrm{~g} . \mathrm{L}^{-1}(25 \mathrm{~mL})$ \\
$\mathrm{KCl}$ & $0,23 \mathrm{~g} . \mathrm{L}^{-1}(25 \mathrm{~mL})$ \\
\hline
\end{tabular}




\subsubsection{Effet de la salinité}

Les tests sont réalisés, dans un premier temps, en présence de solutions salines sans addition d'ions métalliques, afin d'examiner l'influence de ce paramètre sur le comportement des daphnies. Dans ce cas, les solutions salines utilisées dans les biotests sont constituées en ajoutant à l'eau de dilution des quantités suffisantes de $\mathrm{NaCl}$ devant permettre d'obtenir des salinités égales à 0,42 et $2,45 \mathrm{~g} \cdot \mathrm{L}^{-1}$.

En présence d'ions métalliques, trois concentrations salines ont été étudiées et les conductivités obtenues sont données sur le tableau 2.

Dans ce cas, on maintient constante la concentration de la solution saline et on fait varier la concentration de l'ion métallique.

La salinité est mesurée à l'aide d'un conductimètre «Hydromat», type LN302.

\subsubsection{Effet de la dureté}

La composition de la solution mère (ISO, 1989) est récapitulée sur le tableau 3.

- Comme précédemment, les tests sont réalisés, dans un premier temps, en présence de solutions carbonatées sans addition d'ions métalliques afin d'examiner l'influence de ce paramètre sur le comportement des daphnies. Dans ce cas, la dureté a été modifiée en fonction des quantités de substances contenant $\mathrm{Ca}$ et $\mathrm{Mg}$ dont la composition de la solution est indiquée dans le tableau 3, tout en maintenant constants les rapports $\mathrm{Ca} / \mathrm{Mg}$ et $\mathrm{Na} / \mathrm{K}$. En présence d'ions métalliques,

Tableau 2. Salinité et conductivité des milieux sans métaux lourds. Table 2. Salinity and conductivity of the media without heavy metals.

\begin{tabular}{lccc}
\hline Solutions $\rightarrow$ & I & II & III \\
\hline Salinité $\left(\mathrm{g} . \mathrm{L}^{-1} \mathrm{NaCl}\right)$ & 0,28 & 0,45 & 2,42 \\
Conductivité $\left(\mu \mathrm{S} . \mathrm{cm}^{-1}\right)$ & 0,629 & 0,859 & 3,78 \\
\hline
\end{tabular}

Tableau 3. Composition de la solution mère utilisée lors des tests sur l'effet de la dureté.

Table 3. Composition of the indiluted solution used during the tests on the effect of hardness.

\begin{tabular}{ll}
\hline $\mathrm{NaHCO}_{3}$ & 0,800 g.L \\
$\mathrm{CaCl}_{2}, 2 \mathrm{H}_{2} \mathrm{O}$ & $1,188 \mathrm{~g} \cdot \mathrm{L}^{-1}$ \\
$\mathrm{~K}_{2} \mathrm{SO}_{4}$ & $0,104 \mathrm{~g} \cdot \mathrm{L}^{-1}$ \\
$\mathrm{MgCl}_{2}, 6 \mathrm{H}_{2} \mathrm{O}$ & $0,668 \mathrm{~g} . \mathrm{L}^{-1}$ \\
\hline
\end{tabular}

deux concentrations en carbonates sont édudiées : 250 et $500 \mathrm{mg} \cdot \mathrm{L}^{-1} \mathrm{de} \mathrm{CaCO}_{3}$.

Dans ce cas, on maintient constante la concentration de la solution en carbonates et on fait varier la concentration de l'ion métallique.

La dureté est mesurée suivant la méthode complexométrique à l'E.D.T.A.

- Ions métalliques

Dans tous les tests, les ions métalliques utilisés sont sous forme de sels sulfatés : $\mathrm{Al}_{2}\left(\mathrm{SO}_{4}\right)_{3}, 14 \mathrm{H}_{2} \mathrm{O}$; $\mathrm{CdSO}_{4}, 8 / 3 \mathrm{H}_{2} \mathrm{O} ; \mathrm{Cr}_{2}\left(\mathrm{SO}_{4}\right)_{3}, 15 \mathrm{H}_{2} \mathrm{O} ; \mathrm{CuSO}_{4}, 5 \mathrm{H}_{2} \mathrm{O}$; $\mathrm{NiSO}_{4}, \mathrm{H}_{2} \mathrm{O} ; \mathrm{ZnSO}_{4}, \mathrm{H}_{2} \mathrm{O}$.

Les concentrations en ions métalliques varient de :

- 0,01 à $0,08 \mathrm{mg} . \mathrm{L}^{-1}$ pour $\mathrm{Cd}$

$-0,01$ à $0,18 \mathrm{mg} \cdot \mathrm{L}^{-1}$ pour $\mathrm{Cu}$

$-1,00$ à $10,00 \mathrm{mg} \cdot \mathrm{L}^{-1}$ pour $\mathrm{Ni}$

$-3,00$ à $18,00 \mathrm{mg} \cdot \mathrm{L}^{-1}$ pour $\mathrm{Zn}$

$-3,00$ à $26,00 \mathrm{mg} \cdot \mathrm{L}^{-1}$ pour $\mathrm{Al}$

- 3,00 à 23,00 mg. $\mathrm{L}^{-1}$ pour $\mathrm{Cr}$

\subsection{Méthode de calcul}

Le calcul des $\mathrm{Cl} 50-24 \mathrm{~h}$ et $\mathrm{Cl} 150-48$ h est réalisé à l'aide d'un logiciel utilisant la méthode «Probit», décrite dans l'ouvrage de Finney (1952).

L'analyse statistique des résultats est effectuée à l'aide de tests de comparaison qui font appel à la variance (ANOVA).

\section{Résultats et discussion}

\subsection{Effet de la salinité}

Les résultats obtenus sur l'effet de la salinité, sans addition d'ions métalliques, montrent que la concentration en sels qui inhibe la mobilité de $50 \%$ des daphnies après $24 \mathrm{~h}$ d'exposition est de 2,14 g.L $\mathrm{L}^{-1}$ de $\mathrm{NaCl}$, alors qu'après $48 \mathrm{~h}$, elle diminue à $0,49 \mathrm{~g} \cdot \mathrm{L}^{-1} \mathrm{de} \mathrm{NaCl}$.

Soit : Cl50-24 h : 2,14 g.L-1 de NaCl

$$
\text { Cl50-48 h : 0,49 g.L } \text { - }^{-1} \text { de } \mathrm{NaCl}
$$

En présence d'ions métalliques, les résultats des Cl50 sont récapitulés sur le tableau 4.

Ces résultats font ressortir l'influence de la concentration en sels sur l'inhibition apportée par le métal présent dans le milieu.

La littérature relative à l'influence simultanée de la salinité et des ions métalliques indique une augmentation de la tolérance vis-à-vis de ces ions lorsque la salinité est élevée (Poldoski 1979 : réactif chimique : Cd dans les lacs; réactif biologique : D. magna; Bryant et 
Tableau 4. Détermination des $\mathrm{Cl} 50-24 \mathrm{~h}$ et des Cl50-48 h en fonction de la salinité et pour différentes concentrations en ions métalliques. Table 4. Determination of $\mathrm{Cl} 50-24 \mathrm{~h}$ and $\mathrm{Cl} 50-48 \mathrm{~h}$ as a function of salinity and various metal ions concentrations.

\begin{tabular}{|c|c|c|c|c|}
\hline \multirow[t]{2}{*}{$\mathrm{Cl}$} & \multirow[t]{2}{*}{ Ions métalliques } & \multicolumn{3}{|c|}{ Salinité (g.L-1 $\mathrm{NaCl}$} \\
\hline & & 0,28 & 0,42 & $2,42^{(a)}$ \\
\hline \multirow{6}{*}{$\mathrm{Cl} 50-24 \mathrm{~h}$} & $\mathrm{Al}^{3+}$ & 13,820 & $10,990^{(a)}$ & $5,610^{(a)}$ \\
\hline & $\mathrm{Cu}^{2+}$ & 0,100 & 0,080 & $0,070^{(a)}$ \\
\hline & $\mathrm{Cd}^{2+}$ & 0,0594 & 0,0379 & 0,0068 \\
\hline & $\mathrm{Cr}^{3+}$ & 14,970 & 20,160 & 20,170 \\
\hline & $\mathrm{Ni}^{2+}$ & 5,830 & $9,230^{(a)}$ & 4,600 \\
\hline & $\mathrm{Zn}^{2+}$ & 9,580 & 6,130 & 6,100 \\
\hline \multirow{6}{*}{$\mathrm{Cl} 50-48 \mathrm{~h}$} & $\mathrm{Al}^{3+}$ & 5,990 & 4,260 & $2,520^{(a)}$ \\
\hline & $\mathrm{Cu}^{2+}$ & 0,030 & 0,060 & $0,050^{(a)}$ \\
\hline & $\mathrm{Cd}^{2+}$ & 0,0046 & $0,0109^{(a)}$ & 0,0050 \\
\hline & $\mathrm{Cr}^{3+}$ & 11,530 & 13,810 & 14,290 \\
\hline & $\mathrm{Ni}^{2+}$ & 1,820 & $4,200^{(a)}$ & 1,150 \\
\hline & $\mathrm{Zn}^{2+}$ & 4,600 & $2,000^{(a)}$ & $2,280^{(a)}$ \\
\hline
\end{tabular}

(a) : Différence significative par rapport aux tests dans les conditions de salinité proposées par la norme ISO (1989)

al. $1985 b$ : réactifs chimiques : $\mathrm{Ni}$ et $\mathrm{Zn}$; réactifs biologiques : Corophium volutator et Macoma balthica ; Persoone et al. 1989 : réactif biologique : Daphnia magna ; réactifs chimiques : Ni et $\mathrm{Zn}$. On note, par contre, dans la présente étude que pour $\mathrm{Al}, \mathrm{Cu}, \mathrm{Cd}$ et $\mathrm{Zn}$ l'effet de toxicité du métal en $24 \mathrm{~h}$ est plus important à mesure que la salinité du milieu augmente (Figs 1 à 4). Par exemple pour le $\mathrm{Cd}$, la $\mathrm{Cl} 50-24$ h passe de 0,0594 mg. $L^{-1}$ pour une salinité de $0,28 \mathrm{~g} . \mathrm{L}^{-1}$ de $\mathrm{NaCl}$ à $0,0068 \mathrm{mg} . \mathrm{L}^{-1}$ pour une salinité de $2,42 \mathrm{~g} . \mathrm{L}^{-1}$. De même, pour $\mathrm{Al}, \mathrm{Zn}$ et $\mathrm{Cu}$, les $\mathrm{Cl} 50-24 \mathrm{~h}$ de 13,$82 ; 9,58$ et $0,1 \mathrm{mg} \cdot \mathrm{L}^{-1}$ pour une salinité de 0,28 diminuent respectivement à des valeurs de 5,$61 ; 6,1$ et 0,07 mg.L ${ }^{-1}(\mathrm{p}<$ $0,05)$ pour une salinité de 2,42 g.L.1.

On observe pour le chrome que les $\mathrm{Cl} 50-24 \mathrm{~h}$ ont plutôt tendance à augmenter lorsque la salinité s'accroît (Fig. 5). Ceci peut probablement s'expliquer par la formation de complexes qui tendraient à réduire la toxicité du métal. Des essais sont en cours pour vérifier cette hypothèse.

Plusieurs hypothèses ont été formulées quant au possible effet de la salinité sur la toxicité des composés xénobiotiques : variations de la force ionique, inhibition compétitive avec les cations et anions, effets osmotiques directs sur les organismes (Lee 1973, Dalla
Venizia et al. 1980, Moore \& Ramamoothy 1983, Riedel 1985).

Selon Randet \& Petrocelli (1984), l'effet de la salinité dépend tout particulièrement de la nature génétique des biotests utilisés qui déterminent si cet organisme peut s'adapter à une salinité donnée et exprimer alors une tolérance vis-à-vis des toxiques.

Pour $\mathrm{Al}, \mathrm{Cu}$ et $\mathrm{Zn}$, les $\mathrm{Cl} 50-48 \mathrm{~h}$ sont significativement réduites $(\mathrm{p}<0,05)$ quand la salinité passe de 0,28 à 2,42 g.L. ${ }^{-1}$ de $\mathrm{NaCl}$. Le $\mathrm{Cr}$ devient moins toxique pour une salinité supérieure. En effet, les Cl50-48 h sont de 11,53 et $14,29 \mathrm{mg} . \mathrm{L}^{-1}$ pour des salinités égales respectivement à 0,28 et 2,42 g.L. $\mathrm{L}^{-1}$ de $\mathrm{NaCl}$.

Enfin, on note qu'il n'apparât pas de différence significative entre les $\mathrm{Cl} 50-48 \mathrm{~h}$ du $\mathrm{Cd}$ et Ni lorsque la salinité augmente de 0,28 à 2,42 g.L. $\mathrm{L}^{-1}$ (Tableau 4, Figs. $6,7)$. Au bout de ce temps de contact, le phénomène d'osmose tendrait à neutraliser l'action de l'ion métallique par rapport à celle du sel.

Les résultats des tests sur $24 \mathrm{~h}$ nous permettent de proposer ce classement par ordre de toxicité croissante :

$$
\begin{array}{ll}
\mathrm{S}=0,28 \mathrm{~g} \cdot \mathrm{L}^{-1} \mathrm{NaCl} & \mathrm{Cd}>\mathrm{Cu}>\mathrm{Ni}>\mathrm{Zn}>\mathrm{Al}>\mathrm{Cr} \\
\mathrm{S}=0,42 \mathrm{~g} \cdot \mathrm{L}^{-1} \mathrm{NaCl} & \mathrm{Cd}>\mathrm{Cu}>\mathrm{Zn}>\mathrm{Ni}>\mathrm{Al}>\mathrm{Cr} \\
\mathrm{S}=2,42 \mathrm{~g} \cdot \mathrm{L}^{-1} \mathrm{NaCl} & \mathrm{Cd}>\mathrm{Cu}>\mathrm{Ni}>\mathrm{Zn}>\mathrm{Al}>\mathrm{Cr}
\end{array}
$$



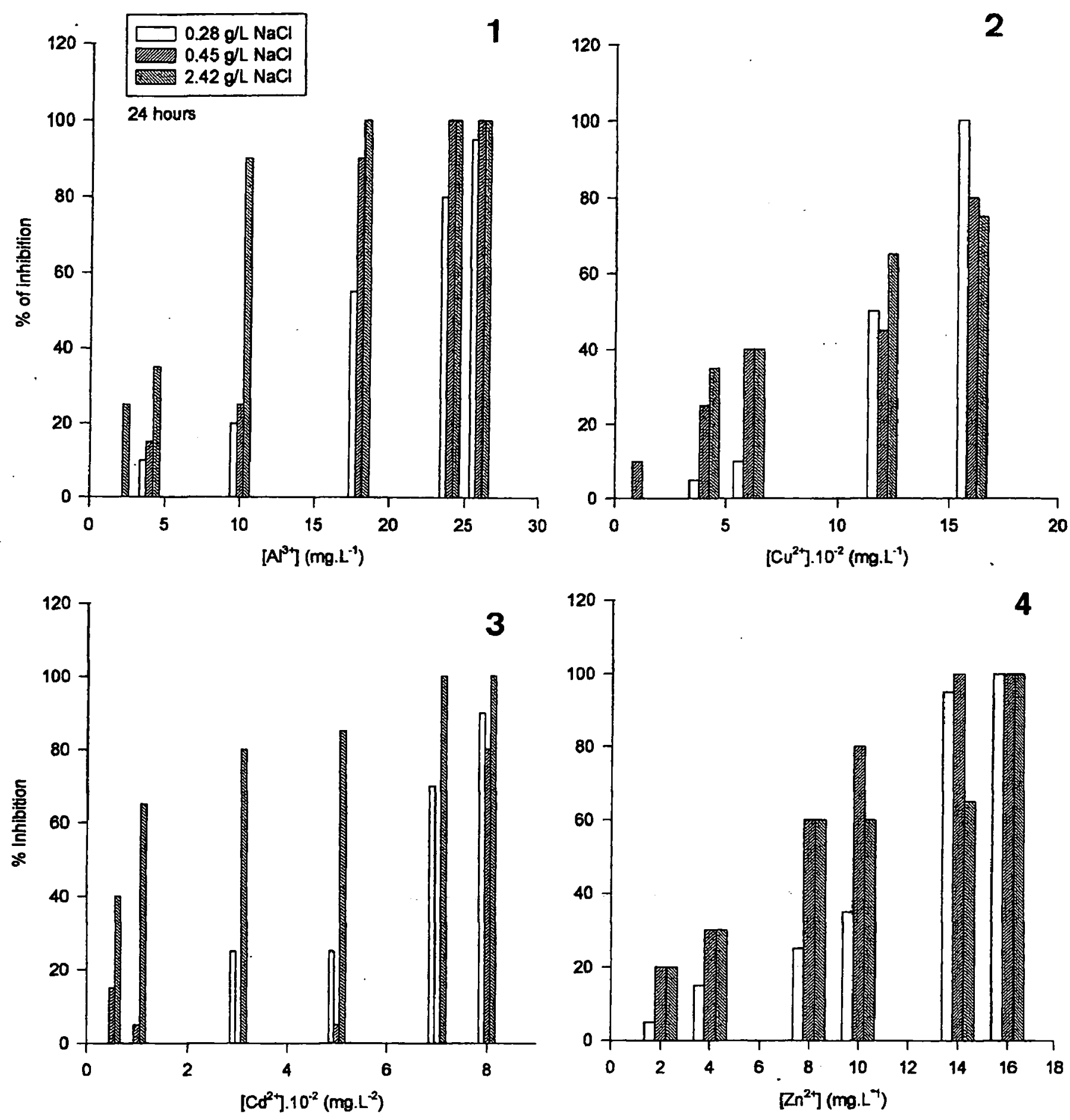

Figs. 1-4. Effet de la salinité sur la toxicité des métaux (Test : 24 heures). 1 : aluminium ; 2 : cuivre ; $3:$ cadmium ; 4 : zinc.

Figs. 1-4. Effect of salinity on metal toxicity (Test : 24 hours). $1:$ aluminium $; 2:$ copper $; 3:$ cadmium $; 4:$ zinc.

\subsection{Effet de la dureté}

En l'absence d'ions métalliques, les résultats indiquent que la $\mathrm{Cl} 50-24 \mathrm{~h}$ et la $\mathrm{Cl} 50-48 \mathrm{~h}$ sont respectivement égales à 488,63 et 267,60 mg.L-1 de $\mathrm{CaCO}_{3}$.

Plusieurs auteurs ont montré que les métaux lourds sont généralement plus toxiques dans une eau douce que dans une eau dure (Abel 1974 : réactifs biologiques : poissons et invertébrés aquatiques : réactifs -chimiques : détergents;; Dave 1978 : réactif biologique : D. magna ; réactif chimique : cadmium ; Muller 1980 : réactif biologique : $D$. magna; réactif chimique : $\mathrm{K}_{2} \mathrm{Cr}_{2} \mathrm{O}_{7}$ ).

Dans la présente étude on observe, en présence d'ions métalliques (Tableau 5), une diminution significative $(\mathrm{p}<0,05)$ de la $\mathrm{Cl} 50-24$ h pour $\mathrm{Al}$ (Fig. 8) de 13,82 à $4,24 \mathrm{mg} \cdot \mathrm{L}^{-1}$ lorsque la dureté passe de 250 à $500 \mathrm{mg} . \mathrm{L}^{-1}$ de $\mathrm{CaCO}_{3}$. De même, pour le $\mathrm{Zn}$ la Cl50$24 \mathrm{~h}$ passe de $9,58 \mathrm{mg} . \mathrm{L}^{-1}$ pour $250 \mathrm{mg} . \mathrm{L}^{-1}$ de $\mathrm{CaCO}_{3}$ à $5,94 \mathrm{mg} . \mathrm{L}^{-1}$ pour $500 \mathrm{mg} . \mathrm{L}^{-1}$. 

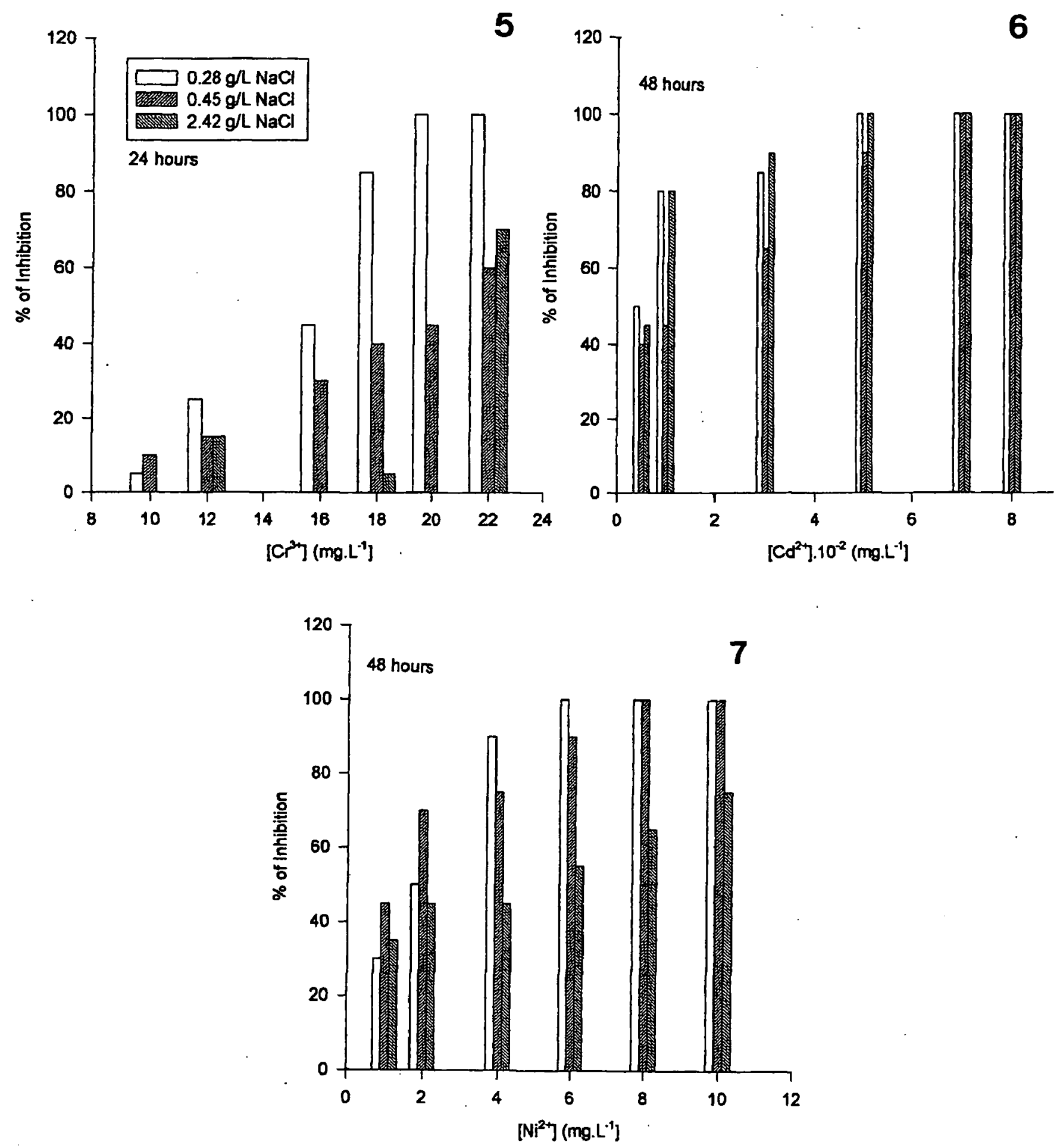

Figs. 5-7. Effet de la salinité sur la toxicité des métaux (Test : 24 heures). 5 : chrome ; 6 : cadmium ; 7 : nickel.

Figs. 5-7. Effect of salinity on metal toxicity (Test : 24 hours). $5:$ chromium ; $6:$ cadmium ; $7:$ nickel.

Des travaux antérieurs (Zitko \& Carson 1976, Carter \& Nicholas 1978, Holcombe \& Andrew 1978) avaient également montré que la dureté de l'eau de dilution avait un effet plus important sur la toxicité du zinc.

Biesinger \& Christensen (1972) ont montré que la toxicité du cuivre est moindre dans un milieu de faible dureté, ce que confirme notre étude.

Pour les autres ions, la diminution n'est pas significative. Pour le Ni (Fig. 9), la $\mathrm{Cl} 50-24$ h diminue de 5,83 à 4,81 mg.L ${ }^{-1}$ alors que pour le Cd (Fig. 10) elle diminue de 0,0594 à $-0,0306 \mathrm{mg} \cdot \mathrm{L}^{-1}$ pour la même variation de la dureté. Des résultats similaires sont obtenus pour le $\mathrm{Cu}$; ils confirment les essais de Gauss et al. (1985) qui ont observé qu'en augmentant la dureté de l'eau jusqu'à $800 \mathrm{mg} . \mathrm{L}^{-1}$, la toxicité du cuivre changeait très faiblement.

Seul le Cr ne semble pas aussi toxique que les autres métaux lorsque la dureté augmente puisqu'on observe, au contraire, une augmentation de la $\mathrm{Cl} 150-24 \mathrm{~h}$ de 14,97 à $18,92 \mathrm{mg} . \mathrm{L}^{-1}$. 
Tableau 5. Effet de la dureté : détermination des Cl50-24 h et des Cl50-48 h.

Table 5. Effect of hardness : determination of the $\mathrm{Cl} 50-24 \mathrm{~h}$ and the Cl50-48 $\mathrm{h}$.

\begin{tabular}{|c|c|c|c|}
\hline \multirow[t]{2}{*}{$\mathrm{Cl}$} & \multirow{2}{*}{$\begin{array}{c}\text { Ions métalliques } \\
\mathrm{xxxx}\end{array}$} & \multicolumn{2}{|c|}{ Dureté (mg.L-1 $\mathrm{CaCO}_{3}$} \\
\hline & & 250 & 500 \\
\hline & $\mathrm{Al}^{3+}$ & 13,82 & $4,2400^{(a)}$ \\
\hline & $\mathrm{Cd}^{2+}$ & 0,0594 & 0,0306 \\
\hline \multirow[t]{5}{*}{ C150-24 h } & $\mathrm{Cu}^{2+}$ & 0,1000 & 0,0500 \\
\hline & $\mathrm{Ni}^{2+}$ & 5,3800 & 4,8100 \\
\hline & $\mathrm{Zn}^{2+}$ & 9,5800 & $5,9400^{(a)}$ \\
\hline & $\mathrm{Cr}^{3+}$ & 14,970 & $18,920^{(a)}$ \\
\hline & $\mathrm{Al}^{3+}$ & 5,9900 & $2,0000^{(a)}$ \\
\hline \multirow[t]{5}{*}{$\mathrm{Cl} 150-48 \mathrm{~h}$} & $\mathrm{Cd}^{2+}$ & 0,0046 & 0,0071 \\
\hline & $\mathrm{Cu}^{2+}$ & 0,0300 & 0,0100 \\
\hline & $\mathrm{Ni}^{2+}$ & 1,8200 & 1,2600 \\
\hline & $\mathrm{Zn}^{2+}$ & 4,6000 & $2,5200^{(a)}$ \\
\hline & $\mathrm{Cr}^{3+}$ & 11,530 & 13,350 \\
\hline
\end{tabular}

(a) : Différence significative $(\mathrm{p}<0,05)$ entre essais à 250 et $500 \mathrm{mg} \cdot \mathrm{L}^{-1} \mathrm{CaCO}_{3}$.

Ces résultats nous permettent de penser que les cations bivalents tendent à augmenter la toxicité de certains ions métalliques $(\mathrm{Al}, \mathrm{Zn})$ pour les deux duretés de tests, même si cette action est moins significative pour $\mathrm{Ni}$ et $\mathrm{Cd}$.

Deux hypothèses peuvent être émises :

- une interaction compétitive avec les cations et, plus particulièrement le calcium, peut être la cause d'une augmentation de la toxicité, entraînant une hausse de l'activité ionique du métal ;

- la formation de complexes insolubles carbonatesmétaux lourds ; cette hypothèse n'est cependant plus valable si ces complexes ne sont pas ingérés par les daphnies (Andrew et al. 1977).

Indépendamment de son interaction avec les métaux, le Ca peut lui-même exercer des effets importants sur l'état physiologique des daphnies. Winner \& Gauss (1986), Paulauskis \& Winner (1988) indiquent par exemple, qu'en plus de l'effet antagoniste entre le $\mathrm{Zn}$ et le $\mathrm{Ca}$ en solution, une proportion indéterminée de la diminution de la toxicité du zinc peut résulter des différences dans l'état physiologique des bioindicateurs.
Les résultats des tests sur 24 h nous permettent de proposer ce classement par ordre de toxicité décroissante :

Dureté $250 \mathrm{mg} \cdot \mathrm{L}^{-1} \mathrm{CaCO}_{3} \mathrm{Cd}>\mathrm{Cu}>\mathrm{Ni}>\mathrm{Zn}>\mathrm{Al}>\mathrm{Cr}$

Dureté $500 \mathrm{mg} . \mathrm{L}^{-1} \mathrm{CaCO}_{3} \mathrm{Cd}>\mathrm{Cu}>\mathrm{Ni}>\mathrm{Al}>\mathrm{Zn}>\mathrm{Cr}$

\section{Conclusion}

Cette étude sur les daphnies nous a permis d'examiner d'une part, les effets conjugués de la salinité et des ions métalliques et d'autre part, les effets conjugués de la dureté et des ions métalliques.

Ainsi, l'influence simultanée du couple salinité-ions métalliques indique, d'une manière générale, une diminution de la tolérance de ces ions lorsque la salinité augmente.

Plusieurs hypothèses ont été formulées quant aux possibles effets de la salinité sur la toxicité des composés xénobiotiques : variation de la force ionique, inhibition compétitive avec les cations et anions, effets osmotiques directs sur les organismes.

Concernant l'influence simultanée du couple duretéions métalliques, nos résultats nous permettent de penser que les cations bivalents tendent à augmenter signi- 

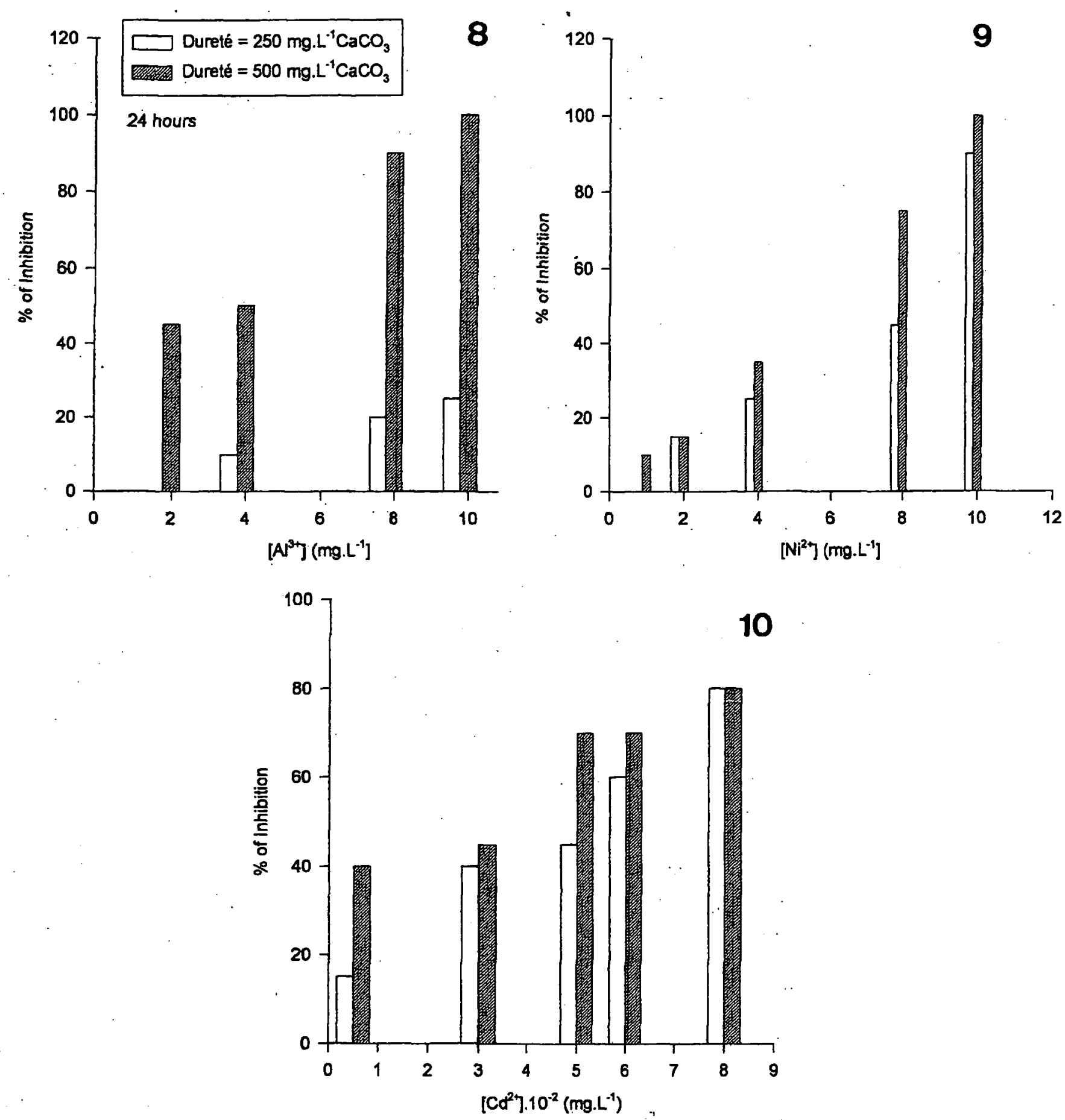

Figs. 8-10. Effet de la dureté sur la toxicité des métaux (Test : 24 heurés). $8:$ aluminium ; $9:$ nickel ; $10:$ cadmium. Figs. 8-10. Effect of hardness on metal toxicity (Test : 24 hours). $8:$ aluminium ; $9:$ nickel ; $10:$ cadmium.

ficativement la toxicité de certains ions métalliques $(\mathrm{Al}, \mathrm{Zn})$ même si cette action est moins significative pour le Ni et le Cd.

- Indépendamment de son interaction avec les métaux, le Ca peut lui-même exercer des effets importants sur l'état physiologique des Daphnies.

Deux hypothèses ont été émises :

- une interaction compétitive avec les cations et, plus particulièrement le calcium, peut causer une élévation de la toxicité, et entraîner une augmentation de l'activité ionique du métal ;

- la formation de complexes insolubles carbonatesmétaux lourds ; cette hypothèse n'est plus acceptable lorsque ces complexes ne sont pas plus toxiques que la forme libre du métal.

\section{Travaux cités}

Abel P.D. 1974. - Toxicity of synthetic detergent to fish and aquatic invertebrates. J. Fish. Biol., $6: 279-298$. 
Andrew R.W., Beisinger K.E. \& Glass G.E. 1977. - Effects of inorganic complexing on the toxicity of copper to Daphnia magna. Water Res., 11 : 309-315.

Berling R. \& Dave G. 1984. - Acute toxicity of chromate DDT, PCP, TPBS and zinc to Daphnia magna cultured in hard and soft water. Bull. Environm. Contam. Toxicol., $33: 63-68$.

Biesinger K.E. \& Christensen M.G. 1972. - Effect of various on survival, growth, reproduction and metabolism of Daphnia magna. J. Fish Res. Bd. Canada, 29 : 1691-1700.

Bryant V., New Bery D.M. \& Campbell R. 1985. - Effect of temperature and salinity on the toxicity of nickel and zinc to tow estuarine invertebrates. Mar. Ecol. Prog. Ser., 24 : 139-153.

Carter J.G.T. \& Nicholas W.L. 1978. - Uptake of zinc by the aquatic larvae of Simulium ornatipes. Aust. J. Mar. Freshwater Res., 29 : 299-309.

Dalla Venezia L., Fossato V. \& Scarfi S. 1980. - Combined effects of dodecylbenzene sulfonate and low salinities on Tisbe bulbisetosa. Prog. Water Technol., 12 : 109-117.

Dave G. 1978. - An annotated literature survey of methods for determination of effects and fate of pollutants in aquatic environment, Blanck H., Dave G. \& Gustafsson K. (eds), Swedish Environment, SNV PM, $1050 \mathrm{p}$.

De Lisle P.F. \& Roberts M.H. 1988. - Osmoregulation in the estuarine mysid, Mysidopsis bahia Molenock : comparaison with other Mysid species. Comp. Biochem. Physiol., 88A : 369-372.

Erickson R.J., Benoit D.A., Mattson V.R., Nelson H.P. \& Leonard E.N. 1996. - The effects of water chemistry on the toxicity of coper to flathead minnows. Environmental Toxicology and Chemistry, 15, 2 : 181-183.

Finney D.J. 1952. - Probit analysis. Cambridge Univ. Press. XIV, London.

Gauss J.D., Woods P.E., Winner R.W. \& Skillings S.H. 1985. Acute toxicity of copper to three live stages of Chironomus tentans as affected by water hardness and alkalinity. Environ. Pollut. Ser. A. Ecol. Biol., 37 : 149-157.

Holcombe G.W. \& Andrew R.W. 1978. - The acute toxicity of zinc to rainbow and brook trout : Comparaisons in hard and soft water. EPA - 600/3 - 78 - 094, U.S., Environmental Protection Agency, Duluth, Minnesota $3: 78$.

ISO (International Organization of Standardization) 1989. - Determination of the inhibition of the mobility of Daphnia magna Strauss (Cladocera Crustacea). $634: 189$ (E), 1-7.

Lee D.R. 1973. - Reference toxicants in quality control of aquatic bioassay criteria. Water Res., 7 : 1525-1546.

Moore J.W. \& Ramamoorthy S. 1983. - Heavy metals in natural waters. - Applied monitoring and impact assessment, Springer Verlag, New-York : $268 \mathrm{p}$.

Muller H.G. 1980. - Acute toxicity of potassium dichromate to Daphnia magna as function of the quality. Bull. Environ. Contam. Toxicol., 25 : 113-117.
Paulauskis J.D. \& Winner R.W. 1988. - Effects of water hardness and humic acid on zinc toxicity to Daphnia magna Straus. Aquat. Toxicol., 12 : 273-290.

Persoone G., Van de Vel A., Van Steertegem M. \& De Nayer B. 1989. - Predictive value of laboratory tests with aquatic invertebrates influence of experimental conditions. Aquatic Toxicol., $14: 149-166$.

Poldoski J.E. 1979. - Cadmium bioaccumulation assays. Their relationship to various ionic equilibria in lake superior. Waters Environ. Sci. Technol., 13 : 701-706.

Rand G.M. \& Petrocelli S.R. 1984. - Fundamentals of aquatic toxicology : methods and applications. Hemisphere Publishing Corporation, Washington : $666 \mathrm{p}$

Riedel G.F. 1985. - The relationship between chromium (VI) upta$\mathrm{ke}$, sulfate uptake and chromium (VI) toxicity in the estuarine diatom. Thalassiosira pseudonana. Aquatic Toxicol., 7 : 191-204.

Semsari S. 1992. - Impact écotoxicologique du chrome trivalent et des sulfures présents dans les effluents de tannerie : Application à l'unité de tannerie-mégisserie de Rouiba (Alger). Thèse de magister, Université des Sciences et de la Technologie Houari Boumédiène, Alger : $156 \mathrm{p}$.

Semsari S. \& Gaïd A. 1993. - Evaluation de la toxicité de quelques métaux lourds à l'aide du test daphnie. La Tribune de l'eau, septoct., $5: 35-43$.

Sprague J.B. 1985. - Factors that modify toxicity in fundamentals of aquatic toxicology, Rand G.M. \& Petrocelli S.R. (eds), Washington : 124-163.

Sunda W.G., Engel D.W. \& Thuotte R.M. 1978. - Effect of chemical speciation on toxicity of cadmium to grass shrimp Palaemonetes pagio: Importance of free cadmium ion. Environ. Sci. Technol., $12: 409-413$.

Theed H. 1980. - Physiological response of estuarine animals to cadmium pollution. Helgol. Wiss. Meeresunters, 33 : 26-35.

Voyer R.A. \& Mc Govern D.G. 1991. - Influence of constant and fluctuating salinity on responses of Mysidopsis bahia exposed to cadmium in a life-cycle test. Aquat. Toxicol., 19:215-230.

Winner R.W. 1986. - Bioaccumulation and toxicity of copper as affected by interaction between humic acid and water hardness. Aquat. Toxicol., $8: 281-293$.

Winner R.W. \& Gauss J.D. 1986. - Relationship between chronic toxicity and bioaccumulation of coper, cadmium and zinc as affected by water hardness and humic acid. Aquat. Toxicol., 8 : 149-161.

Zitko P. \& Carson W.G. 1976. - A mechanism of the effects of water hardness on the lethality of heavy metals to fish. Chemosphere, $5: 299-303$. 